0.2: $0.1-0.4, \mathrm{p}=0.0002$; males $>50$ yrs vs females $>50 \mathrm{yrs}$, $0.4: 0.2-0.9, \mathrm{p}=0.0323)$, running pace $(6-<7 \mathrm{~min} / \mathrm{km}$ vs $8-<9$ $\mathrm{min} / \mathrm{km}, 0.4: 0.2-0.8, \mathrm{p}=0.0062 ; 7-<8 \mathrm{~min} / \mathrm{km}$ vs $8-<9 \mathrm{~min} /$ $\mathrm{km}, 0.5: 0.3-0.9, \mathrm{p}=0.0299$ ) and route (up vs. down, 0.5: $0.3-0.8, \mathrm{p}=0.0046)$.

Conclusion Older females, slower runners and route (down run) were independent factors associated with higher risk of serious/life-threatening MEs in a $90 \mathrm{~km}$ ultramarathon. These data will form the basis to design and implement prevention programs to manage risk of sltMEs at these events.

\section{PROMOTION OF PARA ATHLETE WELL-BEING IN SOUTH AFRICA (THE PROPEL STUDIES), PART I: PROFILES AND PREVALENCE OF PSYCHOLOGICAL DISTRESS}

\begin{abstract}
1,2 Marelise Badenhorst, 1,2,3 Phoebe Runciman, 1,2 James Craig Brown, 1,2 Wayne Derman. ${ }^{1}$ Institute of Sport and Exercise Medicine (ISEM), Division of Orthopaedic Surgery, Department of Surgical Sciences, Faculty of Medicine and Health Sciences, Stellenbosch University, Cape Town, South Africa; ${ }^{2}$ International Olympic Committee (IOC) Research Centre, Cape Town, South Africa; ${ }^{3}$ Department of Sport Science, Faculty of Education, Stellenbosch University, Cape Town, South Africa
\end{abstract}

\subsection{6/bjsports-2021-IOC.68}

Background There is a paucity of research investigating the mental health profiles of para athletes globally, especially in South Africa.

Objective To describe the mental health profiles of a representative sample of para athletes in South Africa.

Design Descriptive, cross-sectional survey.

Setting National to international level athletes competing in the 2019 National Championships for athletes with impairments.

Patients (or Participants) A total of 124 athletes (93 males; 31 females) with a mean age $26.7( \pm 9.2)$.

Interventions (or Assessment of Risk Factors) Between-group differences were analysed using the Mann-Whitney $U$ test or one-way ANOVA. On completion of the questionnaires, all athletes were given information about mental health support services.

Main Outcome Measurements Mental health was measured with the Trait component of the State/Trait Anxiety Inventory (STAI) and the Kessler Psychological Distress Scale (K-10).

Results The mean score for the STAI was 39.2 ( \pm 9.3$)$ units. A quarter $(25 \%)$ of all athletes scored 45 and above, in line with scores of patients with a psychological/psychiatric diagnosis. On average, females $(35.9 \pm 8.7)$ had better STAI scores than males $(40.3 \pm 9.3 ; p=0.02)$ and married $(32.6$ $\pm 8.9)$ athletes had better scores than single (40.2 \pm 8.9 ; $p=0.002)$ athletes. The mean $\mathrm{K}-10$ questionnaire score was $20.4( \pm 6.4)$ units, with no gender difference. However, K10 scores were significantly higher in single than in married athletes $(U=415.5 ; p=0.002)$. Using a cut-off score of $\geq 28$ and $\geq 16$ on the $\mathrm{K}-10,12.2 \%$ and $76.4 \%$ of athletes had symptoms of distress, respectively. Neither scale was associated with level of competition, sport code or impairment type. The K-10 and STAI scores were highly correlated $\left(r_{s}=0.64, p<0.001\right)$.

Conclusions The study found high rates of psychological distress among para athletes, which is an important first step towards the development of prevention strategies. There is a need to further understand and identify mechanisms affecting mental health in this population.

\section{3 \\ PROMOTION OF PARA ATHLETE WELL-BEING IN SOUTH AFRICA (THE PROPEL STUDIES), PART II: IDENTIFICATION OF SLEEP-ASSOCIATED RISK FACTORS}

1,2Wayne Derman, 1,2,3 Phoebe Runciman, 1,2 James Craig Brown, 1,2 Marelise Badenhorst. ${ }^{1}$ Institute of Sport and Exercise Medicine (ISEM), Division of Orthopaedic Surgery, Department of Surgical Sciences, Faculty of Medicine and Health Sciences, Stellenbosch University, Cape Town, South Africa; ${ }^{2}$ International Olympic Committee (IOC) Research Centre, Cape Town, South Africa; ${ }^{3}$ Department of Sport Science, Faculty of Education, Stellenbosch University, Cape Town, South Africa

\subsection{6/bjsports-2021-IOC.69}

Background Good sleeping habits are necessary for optimal practice and performance, as well as for athlete health. Although the sleep characteristics of elite athletes are well described, research is limited on the sleep profile of similarlevel para athletes.

Objective To evaluate sleep quality, sleepiness and chronotype of para athletes in South Africa.

Design Descriptive, cross-sectional survey.

Setting National to international level para athletes competing in the 2019 National Championships.

Patients (or Participants) A total of 124 athletes (93 males; 31 females) with a mean age $26.7( \pm 9.2)$.

Interventions (or Assessment of Risk Factors) Chi-square, with Fisher's exact tests were used to evaluate differences in sleep latency, efficiency, daytime dysfunction, sleep duration, chronotype and sleepiness between 'good' and 'poor' quality sleep groups.

Main Outcome Measurements Pittsburgh Sleep Quality Index (PSQI), the Epworth Sleepiness scale and Morningness-Eveningness Questionnaire (MEQ-SA).

Results Fifty-eight percent (58\%) of athletes identified as morning types, while $38 \%$ identified as intermediate types. Forty-eight percent (48\%) were classified as having 'good' and the remainder as having 'poor' sleep quality. Moderate to severe daytime sleepiness was present in $30 \%$ of athletes. Thirty percent $(30 \%)$ reported sleep duration of 5-6 hours per night, while 5\% slept less than 5 hours. Morning types were significantly associated with the 'good' quality sleep group $(\mathrm{p}<0.001, \mathrm{~V}=0.35)$ and the 'sleepiness' group were associated with the 'poor' quality sleep group $(p=0.04$, $\mathrm{V}=0.19$ ). Additionally, athletes with 'poor' sleep quality were associated with shorter sleep duration $(\mathrm{p}<0.001, \mathrm{~V}=.63)$, greater sleep latency $(\mathrm{p}<0.001, \mathrm{~V}=.62)$, lower sleep efficiency $(\mathrm{p}=0.001, \mathrm{~V}=.45)$, greater daytime dysfunction $(\mathrm{p}<0.001$, $\mathrm{V}=.40)$ and greater sleep disturbances $(\mathrm{p}<0.001, \mathrm{~V} .34)$.

Conclusions The majority of athletes in this study presented with poor sleep quality. These findings demonstrate a need to identify, address and prevent possible mechanisms affecting poor sleep quality in this population.

\section{PROMOTION OF PARA ATHLETE WELL-BEING IN SOUTH AFRICA (THE PROPEL STUDIES), PART III: FACTORS ASSOCIATED WITH MENTAL HEALTH}

\footnotetext{
${ }^{1,2}$ Marelise Badenhorst, ${ }^{1,2,3}$ Phoebe Runciman, 1,2 James Craig Brown, 1,2 Wayne Derman ${ }^{1}$ Institute of Sport and Exercise Medicine (ISEM), Division of Orthopaedic Surgery, Department of Surgical Sciences, Faculty of Medicine and Health Sciences, Stellenbosch University, Cape Town, South Africa; ${ }^{2}$ International Olympic Committee (IOC) Research Centre, Cape Town, South Africa; ${ }^{3}$ Department of Sport Science, Faculty of Education, Stellenbosch University, Cape Town, South Africa
}

10.1136/bjsports-2021-IOC.70 
Background Para athletes may have specific mental health challenges that, together with the demands of high-level sports performance, could put them at risk for mental health disorders.

However, research in this population is limited.

Objective To investigate factors associated with mental health in South African para athletes.

Design Descriptive, cross-sectional survey.

Setting National to international level athletes competing in the 2019 National Championships.

Patients (or Participants) A total of 124 athletes (93 males; 31 females) with a mean age $26.7( \pm 9.2)$ were included in the study.

Interventions (or Assessment of Risk Factors) Demographic, medical history and sleep-related variables were included in bivariate analyses to assess their association with mental health. Between-group differences were analysed using the Mann-Whitney U or T-tests. Variables significantly associated in the bivariate analyses were included in multiple regression analyses for mental health.

Main Outcome Measurements Mental health was measured with the State/Trait Anxiety Inventory (STAI) and the Kessler Psychological Distress Scale (K-10 Questionnaire). Sleep quality, sleepiness and chronotype were measured with the Pittsburgh Sleep Quality Index (PSQI), the Epworth Sleepiness scale and Morningness-Eveningness Questionnaire (MEQ-SA).

Results The model explained $40 \%$ of the variance in mental health $(\mathrm{F}=12.04, \mathrm{p}<0.001)$ in these athletes. Compared to athletes with 'good' sleep quality, K-10 and STAI scores were significantly higher (indicating poorer mental health) in athletes with 'poor' sleep quality $(U=2.6, p<0.001 ; \mathrm{t}(116.8)=-$ $4.30, \mathrm{p}<0.001)$. 'Poor' sleep quality (B:0.8; 95\% CI 0.4 to 1.3), moderate to severe daytime sleepiness (B:4.2; 95\% CI 1.1 to 7.3 ) intermediate (B:3.5 95\% CI 0.4 to 6.6 ) and evening chronotypes (B:12.0 95\% CI 5.0 to 19.1), the presence of allergies (B:3.9 95\% CI 0.1 to 7.6) and male gender (B:3.3 $95 \%$ CI 0.1 to 6.5 ) were most strongly associated with high STAI scores.

Conclusions This study has identified novel factors associated with poor mental health in elite para athletes. As some of these factors are modifiable, further research towards prevention strategies is warranted.

\section{SUICIDAL IDEATION AMONG ELITE ATHLETICS ATHLETES: CROSS-SECTIONAL STUDY OF ASSOCIATIONS WITH SEXUAL AND PHYSICAL ABUSE VICTIMIZATION AND PSYCHOLOGICAL RESOURCEFULNESS}

\footnotetext{
1,2Toomas Timpka, ${ }^{1,2}$ Armin Spreco, ${ }^{1,3}$ Örjan Dahlström, ${ }^{1,2}$ Jenny Jacobsson, 'Jan Kowalski, 1,2,4 Victor Bargoria, ${ }^{5}$ Margo Mountjoy, ${ }^{2}$ Carl Göran Svedin. ' Athletics Research Center, Linköping University, Linköping, Sweden; ${ }^{2}$ Department of Medical and Health Sciences, Linköping University, Linköping, Sweden; ${ }^{3}$ Department of Behavioural Sciences and Learning, Linköping University, Linköping, Sweden; ${ }^{4}$ Department of Orthopaedics and Rehabilitation, Moi University, Eldoret, Kenya; ${ }^{5}$ Department of Family Medicine, McMaster University, Hamilton, Ontario, Canada
}

\subsection{6/bjsports-2021-IOC.71}

Background Physical and sexual abuse victimization have been connected with negative effects on health long after the abuse has stopped. When athletes encounter situations of defeat and entrapment, the likelihood that suicidal ideation will emerge is increased in situations when negative motivational elements are present, for instance, at low levels of social support.
Objective To examine associations between suicidal ideation and sexual and physical abuse among active and recently retired elite Athletics (track and field) athletes.

Design Cross-sectional study.

Setting Swedish elite Athletics.

Participants Athletes $(n=402)$ who had been selected for a Swedish Athletics team for international competitions between 2011 and 2017.

Assessment of Risk Factors Lifetime experience of sexual and physical abuse, athlete personal and sociodemographic characteristics, Athletics participation, sense of coherence (SOC-13), and coping strategies (Brief Cope).

Main Outcome Measurements Suicidal ideation and 1-year period prevalence of non-sports injury.

Results 192 athletes (47.8\%) returned complete data sets. The prevalence of suicidal ideation was $15.6 \%$ (males $17.4 \%$; females 14.2\%) and the non-sports injury prevalence was 8.0\% (males 11.6\%; females 5.7\%). Suicidal ideation was among females (Nagelkerke $R^{2}=0.33$ ) associated with sexual abuse victimization (Odds ratio (OR) 5.94, 95\% Confidence interval (CI) $1.42-24.90 ; P=0.015)$ and lower sense of coherence (OR 0.90, CI 0.85-0.96; $P=0.001)$. Among males $\left(R^{2}=0.25\right)$, suicidal ideation was only associated with use of behavioural disengagement for coping (OR 1.51, CI 1.181.95; $P=0.001)$. Non-sports injury prevalence was among females $\left(R^{2}=0.23\right)$ associated with sexual abuse victimization (OR 8.61, CI $0.02-0.90 ; P=0.039$ ) and participation in an endurance event (OR 7.37, CI 1.11-48.90; $P=0.039$ ), while among males $\left(R^{2}=0.11\right)$ only having immigrant parents (OR, 5.67, CI $1.31-24.45 ; P=0.020)$ was associated with having sustained an injury outside sports.

Conclusions Given that about one out of six athletics athletes had experienced suicidal ideation, the present results warrant and can be used in suicide prevention among elite athletes.

\section{ATHLETE HEALTH MONITORING IN PARALYMPIC ATHLETES: A 52-WEEK PROSPECTIVE STUDY}

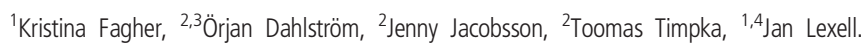
'Department of Health Sciences, Rehabilitation Medicine Research Group, Lund University, Lund, Sweden; '2Department of Medical and Health Sciences, Athletics Research Center, Linköping University, Linköping, Sweden; ${ }^{3}$ Department of Behavioural Sciences and Learning, Linköping University, Linköping, Sweden; ${ }^{4}$ Department of Neuroscience, Rehabilitation Medicine, Uppsala University, Uppsala, Sweden

\subsection{6/bjsports-2021-IOC.72}

Background Sports-related injuries and illnesses in Paralympic sport is a growing concern, but knowledge about the aetiology and risk factors is limited.

Objective To describe the annual incidence of injuries and illnesses among Paralympic athletes and to assess risk factors.

Design Prospective cohort study.

Setting Paralympic athletes

Patients (or Participants) 107 Swedish Paralympic elite athletes with vision, physical and intellectual impairments, active in 19 para sports.

Interventions (or Assessment of Risk Factors) The athletes were asked to weekly report over 52 weeks the annual incidence of injuries, illnesses and quality of health in an adapted eHealth application.

Main Outcome Measurements Time to event, incidence rate (IR) and incidence proportion (IP) and risk factors. 